\title{
Century-scale persistence in longitude distribution in the Sun and in silico
}

\author{
J. Pelt ${ }^{1,2}$, I. Tuominen ${ }^{2}$, and J. Brooke $e^{3,4}$ \\ 1 Tartu Observatory, 61602 Tõravere, Estonia \\ e-mail: pelt@aai.ee \\ 2 Astronomy Division, Department of Physical Sciences, PO Box 3000, 90014 University of Oulu, Finland \\ 3 Manchester Computing, University of Manchester, Oxford Road, Manchester, M13 9PL, UK \\ ${ }^{4}$ Department of Mathematics, University of Manchester, Oxford Road, Manchester, M13 9PL, UK
}

Received 26 May 2004 / Accepted 22 October 2004

\begin{abstract}
Using Greenwich sunspot data for 120 years it was recently observed that activity regions on the Sun's surface tend to lie along smoothly changing longitude strips $180^{\circ}$ apart from each other. However, numerical experiments with random input data show that most, if not all, of the observed longitude discrimination can be an artifact of the analysis method.
\end{abstract}

Key words. Sun: activity - Sun: magnetic fields - Sun: sunspots - methods: statistical

\section{Introduction}

The time distribution of sunspot latitudes is well known and the ubiquitous butterfly diagram is often seen in solar research. The same is not true for longitudes. It is still not known how persistent in time different statistical features of longitude distributions are for various activity indicators (see for instance Bai 2003 and references therein). The present paper is inspired by the recent contribution by Berdyugina \& Usoskin (2003, hereafter $\mathrm{BU})$. By using certain data processing techniques they built smooth curves which run along central longitudes of sunspot activity centres. They observed that the distribution of activity centre phases (if computed against mean flow) has a double peak distribution at a high level of confidence (see Fig. 5 in BU). They concluded that there is a centuryscale persistent feature in the sunspot longitude distribution: the local neigbouring maxima of activity tend to lie $180^{\circ}$ apart. They also observed several "flip-flop" events (see Jetsu et al. 1993) and concluded that there is a strong analogy between the Sun and rapidly rotating, magnetically active late-type stars (for an overview of active stars see Tuominen et al. 2002). In metaphoric terms: the Sun has two faces, and as a kind of peculiar Janus it sometimes switches them back and forth.

In this paper we analyse randomly generated distributions to check the possibility that the observed effect in $\mathrm{BU}$ is a result of dependencies and correlations hidden in the statistical method itself. Indeed, we found that it is possible to obtain quite similar double-peak distributions for random data. Consequently, the statistically significant part (or even all) of the effect described in BU can be ascribed to the artifacts of data processing. It is still reasonable to assume that the mean magnetic field of the Sun (at least in the regions of spot formation) is axisymmetric, when the mean is taken over a sufficiently long time.

What follows is a short technical account of the analysis done.

\subsection{Simulations for active longitude distributions}

The strongest argument in BU for the century-scale persistence of active longitudes is given in their Fig. 5 where the two strong and symmetric distribution maxima indicate that sunspot groups tend to concentrate around two centres, $180^{\circ}$ (0.5 in phase) apart.

To check their result and its methodological underpinnings we did some very simple numerical simulations ${ }^{1}$. Instead of using actual observational data we generated artifical random data sets and tried to process the obtained distributions along the same lines as in the original paper. There was no need to simulate all data processing steps performed in BU. It was enough to start from the point where for each Carrington rotation the phases for activity maxima were computed. For simplicity we assume that for every rotation there are at least two activity maxima, each representing a sunspot group concentration.

Each individual simulation run then proceeded as follows. First, for each of the $N=1720$ Carrington rotations with starting times $t_{i}, i=1, \ldots, N$ two fully random and statistically independent phases $\phi_{1}\left(t_{i}\right), \phi_{2}\left(t_{i}\right), i=$ $1, \ldots, N$ were generated. The evenly distributed values $0<=\phi_{1}\left(t_{i}\right)<1$ and $0<=\phi_{2}\left(t_{i}\right)<1$ were then used as

${ }^{1}$ Full source codes of relevant software can be downloaded from http://www. aai.ee/ pelt/soft.htm 


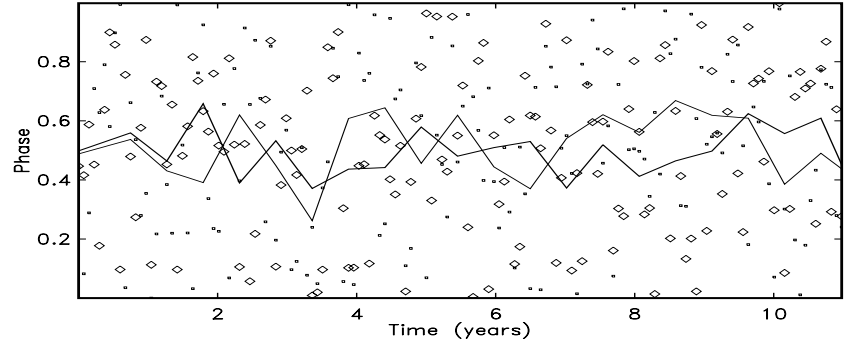

Fig. 1. Fragment of the computer generated phases $\phi_{1}\left(t_{i}\right)$ (diamonds) and $\phi_{2}\left(t_{i}\right)$ (points). Thick line is a half year average of the principal maxima $\left(M_{1}(t)\right)$, thin line is a half year average of the secondary maxima $\left(M_{2}(t)\right)$. The mean curves can cross each other.

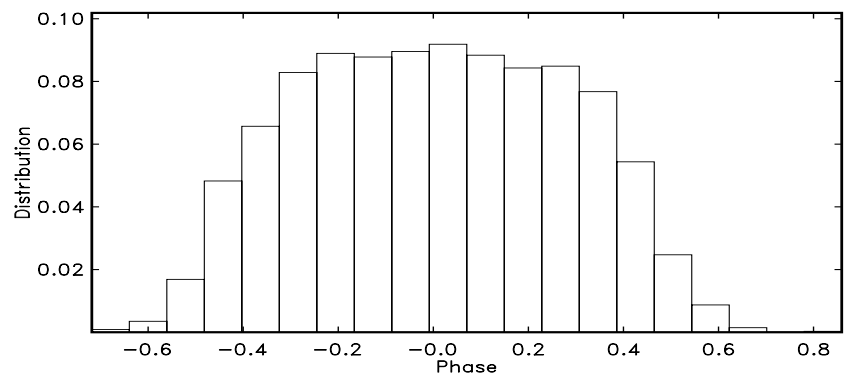

Fig. 2. Distribution of the differences between phases $\phi_{1}\left(t_{i}\right), \phi_{2}\left(t_{i}\right)$ and the half year mean $M^{1}(t)$ for one particular run (first from the series of 100).

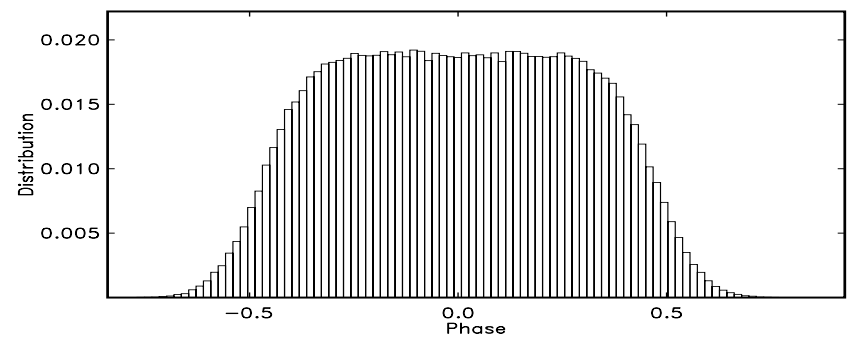

Fig. 3. Mean distribution of the 100 separate runs. The distribution is flat in the centre.

simulated phases for principal and secondary maxima of sunspot density distribution along longitude for particular rotation $t_{i}$.

For both sequences of phases $\phi_{1}$ and $\phi_{2}$ the half year means were calculated and from the mean values, by linear interpolation, the two continuous mean curves $M_{1}(t)$ and $M_{2}(t)$ were built. To get the analogue of Fig. 5 of BU, the distribution of the differences of the generated phases and of the continuous curve $\left(M_{1}(t)\right)$ was computed. In Fig. 1 a short fragment of generated phases together with two running means and in Fig. 2 the corresponding distribution for all differences are plotted. To eliminate the effect of the statistical fluctuations we generated 100 independent distributions and in Fig. 3 the mean distribution of these runs are plotted. The means are uncorrelated and the distribution is approximately flat in its central part.

\subsection{Phase adjustments}

In $\mathrm{BU}$, the original phases of active longitude centers were not used straightforwardly, but were modified. The authors say

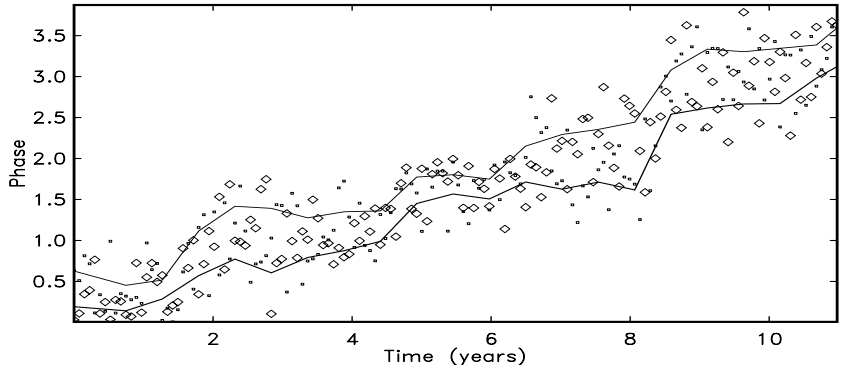

Fig. 4. Two rows of random phases with phase adjustments applied. The upward trend is due to the accumulation of the added full cycles.

"We plot the recovered phases of sunspot clusters versus time and find that regions migrate in phase as rigid structures. When a region reaches $\phi=1$, it appears again near $\phi=0$. In such cases we add an integer to the phase and unfold continuous migration of the regions". Unfortunately the desription of the actual procedure of the described processing step remains somewhat obscure. From Usoskin (2003) we understood that:

- there was a preset constant, say $\delta \phi$.

- when a particular maximum occured at a phase which exceeded $1-\delta \phi$ and when one of the maximum phases for the next rotation was less than $\delta \phi$, then such situations were looked upon as a candidate for a phase jump.

- if the prospective phase jump improved the "continuity" of the assumed migration paths, it was accepted.

It is important to note that the jumps were not constrained by the type of maxima (main or secondary). To ensure continuity of the migration paths the authors of BU allowed swaps between the principal and secondary maxima ("flip-flops").

To avoid any subjective judgement we applied a similar but fully automatic procedure in our simulation program. Because the actual value for $\delta \phi$ (if it ever existed in numerical form) was not communicated to as, we tried multiple values of it. In a "crossing situation" we constructed all possible jumping schemes (no jump at all; largest phase jumps; if both can jump, then smaller phase jumps) and selected in each situation a scheme with the smallest change in phases. Thus, if before the crossing point, the phases for two maxima were $\phi_{1}$ and $\phi_{2}$ and after crossing $\phi_{3}$ and $\phi_{4}$, then the selection criterion was the minimization of $\left|\phi_{1}-\phi_{3}\right|+\left|\phi_{2}-\phi_{4}\right|$. We believe that the actual procedure of the original authors was carried out similarily, even if it was not implemeted as a rigorous algorithm but was done by manual adjusting.

We computed analogously to Fig. 3 the distribution diagrams for several values of $\delta \phi$ in the range $0.05-0.25$. All diagrams had a characteristic bimodal form with two distribution maxima (persistent longitudes). For presentation purposes we give here an example with one particular parameter value $\delta \phi=1 / 18$. This is the most natural value for the case when the histogram method of activity smoothing is used and the number of histogram bins is 18 (see BU). In Fig. 4 we see a short (approximately one solar cycle) fragment of the two mean curves with upward drifting phases. In Fig. 5 the one concrete distribution (effectively the first from a series of 100) is given. 


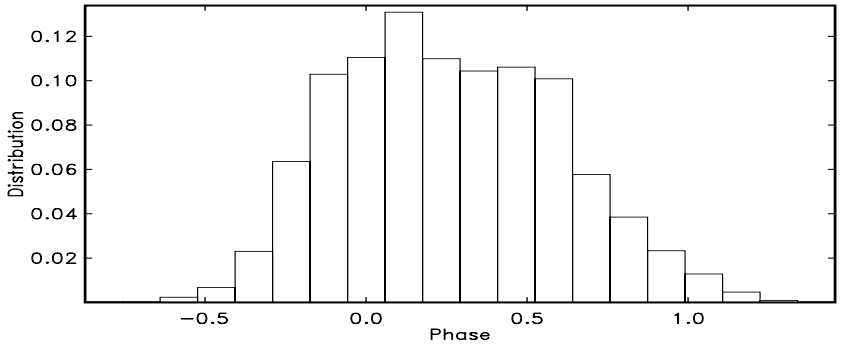

Fig. 5. Distribution of the differences between phases and the mean $M_{1}(t)$ after adjustment. Crossing parameter $\delta \phi=1 / 18$. The bimodal structure of the distribution demonstrates that century-scale persistence of the active longitudes appear even in randomly distributed phases.

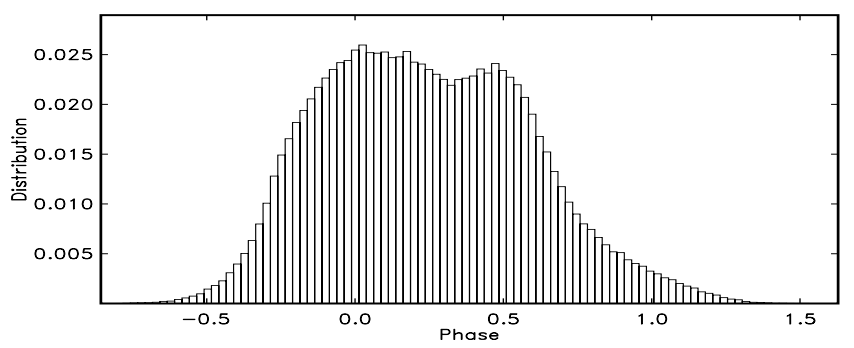

Fig. 6. Mean distribution of the differences for 100 runs. Crossing parameter $\delta \phi=1 / 18$. The bimodality in Fig. 5 is not an exceptional fluctuation.

Figure 6 is the mean distribution of all runs. As we see, the distributions are two peaked.

We started from random and uncorrelated pairs of phases. How it is then possible that there are now certain preferred positions for the random phases? The selection of phase jumping points tends to introduce extra statistical dependencies between neigbouring maxima in different rotations. Some of the large differences $\phi\left(t_{i}\right)-\phi\left(t_{i+1}\right)$ can be excluded by performing jumps for appropriate phases. As a result, the overall mean of differences between sequential phases tends to be less. When these extra correlations and allowed swaps between principal and secondary minima are combined, a double-peaked distribution results.

\subsection{Restrictions from continuity of distributions}

We introduce the notion of circular distance $D\left(\phi_{1}, \phi_{2}\right)$. It is defined as $\min \left(\left|\phi_{1}-\phi_{2}\right|, 1-\left|\phi_{1}-\phi_{2}\right|\right)$ and it takes into account the circular nature of the phases (the point 1 is the starting point 0 for the next turn).

Our simulation model has been unrealistic up to this point. It is possible that both randomly generated phases $\phi_{1}\left(t_{i}\right)$ and $\phi_{2}\left(t_{i}\right)$ are equal or very near to each other. For real data this can be not so. Depending on the smoothing and maximum finding method, there is a certain minimal circular distance $\Delta \phi$ between two phases of maxima. To simulate this kind of more realistic data we generated random phases as was done before. However for each phase pair we computed the circular distance $D\left(\phi_{1}\left(t_{i}\right), \phi_{2}\left(t_{i}\right)\right)$ and pairs whose phases were too close $\left(D\left(\phi_{1}\left(t_{i}\right), \phi_{2}\left(t_{i}\right)\right)<=\Delta \phi\right)$ were excluded from the resulting data

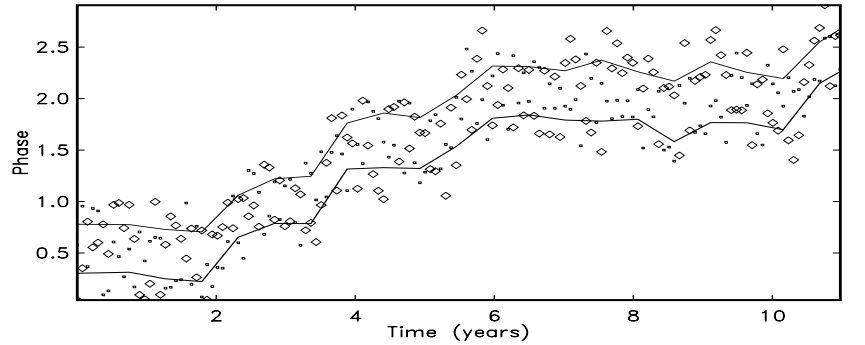

Fig. 7. Fragment of random phases for constrained data. The series of maxima were generated by checking against minimum allowed distance between main and secondary maximum. Crossing parameter $\delta \phi=1 / 18$ and distance parameter $\Delta \phi=0.30$. Adjustments to follow phase drift were also applied.

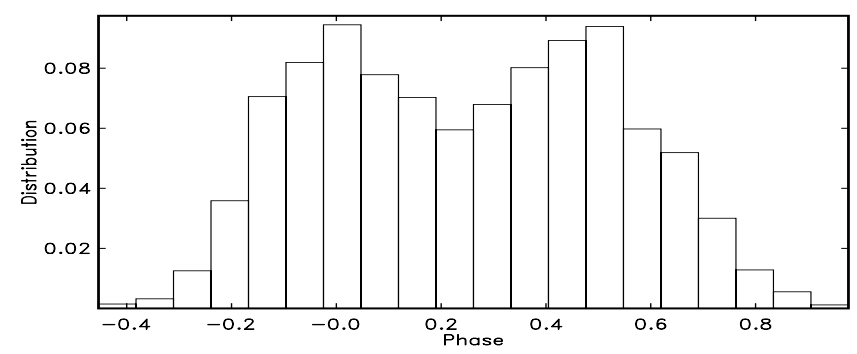

Fig. 8. Phase differences from mean $M_{1}(t)$ for constrained data.

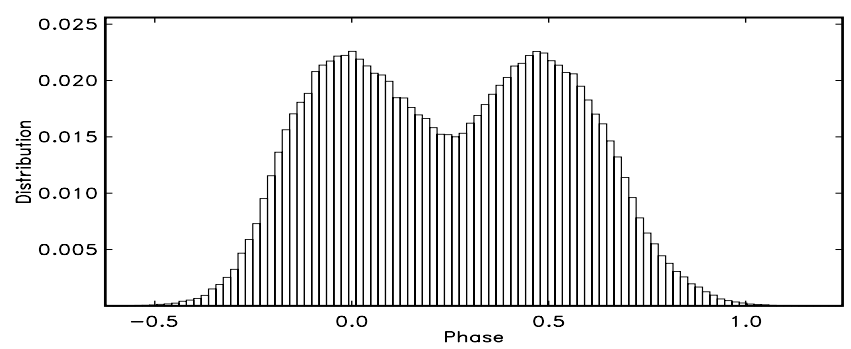

Fig. 9. Mean distribution of the phases for distributions like in Fig. 8.

set. In this way we allow certain statistical dependencies between two phases but phase pairs remain totally independent (from rotation to rotation).

A short fragment of data which is checked against minimal $\Delta \phi$ and with adjusted phases is given in Fig. 7 (crossing parameter $\delta \phi=1 / 18$, distance parameter $\Delta \phi=0.3)$. The tendency of phases to cluster strongly around mean curves is well demonstrated in Fig. 8. The plot of the mean distribution for 100 separate runs (Fig. 9) shows that these are not random fluctuations. It is possible to make the "persistency" effect even stronger if we select certain "optimal" input parameters $\delta \phi$ and $\Delta \phi$.

\subsection{Local correlations}

In the original setup of the generation mechanism for the random data sets, the distributions for all Carrington rotations were assumed to be statistically independent. This is not of course the case for real distributions. It is well known that there are somewhat persistent activity regions on the Sun. If we try to model this kind of local correlation, the separation of the two maxima becomes even more pronounced. 


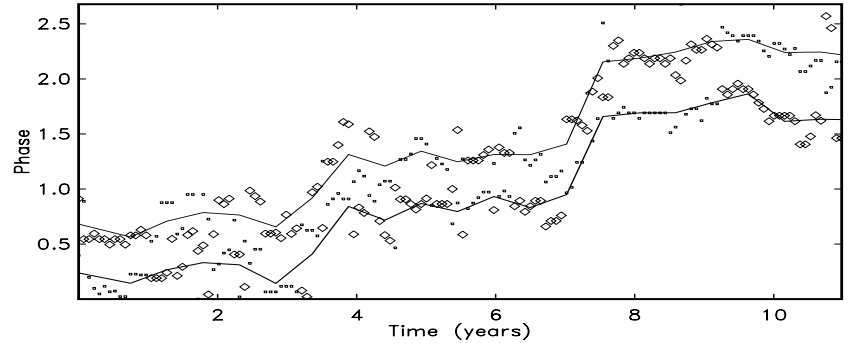

Fig. 10. Two rows of random phases and corresponding mean curves for locally correlated data. Crossing parameter $\delta \phi=1 / 18$, distance parameter $\Delta \phi=0.3$. In this kind of simulation even "flip-flop" events can be seen.

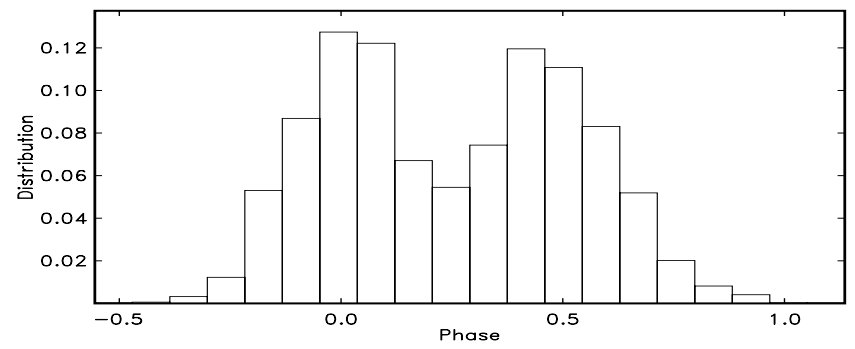

Fig. 11. Phase differences from mean $M_{1}(t)$ for locally correlated data.

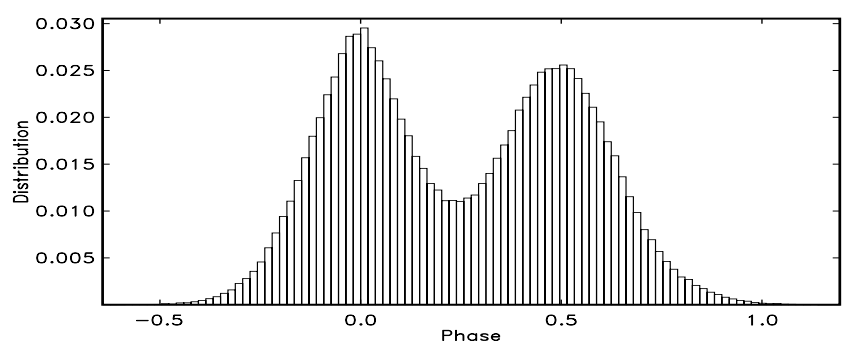

Fig. 12. Mean distribution of the phase differences for distributions like in Fig. 11.

Figures 10 through 12 were obtained using locally correlated data sets. The two-peaked nature is now very pronounced and corresponding plots tend to be quite similar to the plots in the original paper. By fine tuning of the parameters it is possible to get a perfect match. But this is not our goal here.

The local correlations were introduced by using the following generation scheme. For each new phase to be generated, one of the following four possible actions was selected (with equal probability):

- generate totally new phase;

- repeat previous phase;
- add to previous phase a small amount $\delta$;

- subtract from previous phase a small amount $\delta$.

As a result we obtained a sequence of globally uncorrelated (due to the first option) but locally correlated random walk (due to the other options) fragments. We are not arguing here that this is a very good model for solar activity, our aim is just to show that distributions like these in Fig. 5 of $\mathrm{BU}$ can be obtained from locally correlated random sequences and that the correlation can strengthen distribution peaks of preferred longitudes.

\section{Discussion and conclusion}

The analysis above shows that phase distributions of various parameters (maxima in our case) tend to be very sensitive to hidden dependencies and correlations. It is not very easy to see from first glance that for random primary and secondary maxima a phase adjusting to follow "rigid patterns" introduces a significant hidden statistical dependency which shows up as a double peaked distribution of the phase differences.

When we combine all three types of dependencies and correlations, from phase tracking, from constrained phase differences and from real short time correlations, then we easily get significantly bimodal diagrams like Fig. 5 in BU. The flexibility due to the possibility to swap principal and secondary maxima ("flip-flop") and adjust phases ("rigid patterns") allows one to amplify local short-range correlations into century-scale persistent phenomena.

It is important to note that our simulation analysis does not rule out that in principle there can be certain persistent phenomena in sunspot longitude distributions. We have, however, demonstrated that current evidence is not sufficient to conclude this.

Acknowledgements. We are grateful to S. V. Berdyugina and I. G. Usoskin for additional comments about data processing procedures used in the original paper. Part of this work was supported by the Estonian Science Foundation grant No. 4697 and Academy of Finland grant No. 43039.

\section{References}

Bai, T. 2003, ApJ, 585, 1114

Berdyugina, S. V., \& Usoskin, I. G. 2003, A\&A, 1121

Jetsu, L., Pelt, J., \& Tuominen, I. 1993, A\&A, 278, 449

Tuominen, I., Berdyugina, S. V., \& Korpi, M. J. 2002, AN, 323, 367

Usoskin, I. G. 2003, private communication 